\title{
Simulating Progression-Free and Overall Survival for First-Line Doublet Chemotherapy With or Without Bevacizumab in Metastatic Colorectal Cancer Patients Based on Real-World Registry Data
}

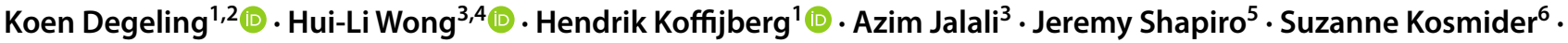 \\ Rachel Wong ${ }^{3,7,8}$ - Belinda Lee $e^{3,4,9} \cdot$ Matthew Burge ${ }^{10}$. Jeanne Tie ${ }^{3,4,6} \cdot$ Desmond Yip $^{11} \cdot$ Louise Nott $^{12}$.

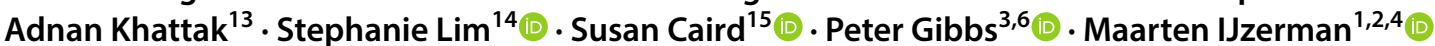

(c) Springer Nature Switzerland AG 2020

\begin{abstract}
Background Simulation models utilizing real-world data have potential to optimize treatment sequencing strategies for specific patient subpopulations, including when conducting clinical trials is not feasible. We aimed to develop a simulation model to estimate progression-free survival (PFS) and overall survival for first-line doublet chemotherapy with or without bevacizumab for specific subgroups of metastatic colorectal cancer (mCRC) patients based on registry data.

Methods Data from 867 patients were used to develop two survival models and one logistic regression model that populated a discrete event simulation (DES). Discrimination and calibration were used for internal validation of these models separately and predicted and observed medians and Kaplan-Meier plots were compared for the integrated DES. Bootstrapping was performed to correct for optimism in the internal validation and to generate correlated sets of model parameters for use in a probabilistic analysis to reflect parameter uncertainty.

Results The survival models showed good calibration based on the regression slopes and modified Hosmer-Lemeshow statistics at 1 and 2 years, but not for short-term predictions at 0.5 years. Modified C-statistics indicated acceptable discrimination. The simulation estimated that median first-line PFS (95\% confidence interval) of $219(25 \%)$ patients could be improved from 175 days (156-199) to 269 days (246-294) if treatment would be targeted based on the highest expected PFS. Conclusions Extensive internal validation showed that DES accurately estimated the outcomes of treatment combination strategies for specific subpopulations, with outcomes suggesting treatment could be optimized. Although results based on real-world data are informative, they cannot replace randomized trials.
\end{abstract}

\section{Introduction}

Clinical outcomes for metastatic colorectal cancer (mCRC) patients have improved substantially over the last 2 decades, partly due to increased efficacy of systemic treatment [1]. Initially, the availability of irinotecan and oxaliplatin made a major contribution to these improved outcomes [2-6]. Subsequently, the addition of bevacizumab, a vascular

Electronic supplementary material The online version of this article (https://doi.org/10.1007/s40273-020-00951-1) contains supplementary material, which is available to authorized users.

Peter Gibbs and Maarten IJzerman contributed equally.

Koen Degeling

koen.degeling@unimelb.edu.au

Extended author information available on the last page of the article endothelial growth factor monoclonal antibody [7-11], was shown to further improve patient survival outcomes. This increase in treatment options, however, also created challenges regarding decisions about which (combination) of these drugs should be used for first-line and subsequent lines of treatment.

Numerous clinical trials have been performed to compare survival following first-line and second-line treatment in mCRC. For example, first-line irinotecan-based doublet chemotherapy [e.g., in combination with fluorouracil and leucovorin (FOLFIRI)] compared with oxaliplatin-based doublet chemotherapy [e.g., in combination with fluorouracil and leucovorin (FOLFOX)] has been studied [12], as well as first- and second-line sequencing of FOLFOX and FOLFIRI [13], and triplet chemotherapy (FOLFOXIRI) [14]. Furthermore, clinical trials have investigated adding bevacizumab to irinotecan- or oxaliplatin-based doublet 


\section{Key Points}

Treatment sequencing complexity in metastatic colorectal cancer (mCRC) is unlikely to be resolved by randomized clinical studies only, indicating an opportunity for simulation models utilizing real-world data (RWD) to provide information on optimal treatment sequencing for specific patient subpopulations.

This study presents the first mCRC simulation model that reflects patient heterogeneity, stochastic uncertainty, and downstream treatment effects based on RWD, to estimate multiple clinical outcomes based on patient and disease characteristics and treatment history.

Results presented show that a simulation utilizing RWD can be used to identify the optimal combination of firstline treatment options for specific mCRC patient subpopulations, demonstrating the potential of such an approach to be extended to optimize treatment sequencing, as well as the value it may have as a decision-support tool.

chemotherapy $[15,16]$ or triplet chemotherapy $[17,18]$, as well as maintenance strategies [19]. However, although preferable, clinical trials cannot be used to evaluate all relevant treatment combinations and their optimal sequencing over multiple lines of treatment, because of the required amount of time and resources and the rate at which new therapies become available.

Further challenges remain regarding treatment sequencing in $\mathrm{mCRC}$ based on patient and disease characteristics and previous treatment outcomes [20,21]. To address these, analyses of clinical trials have stratified efficacy estimates from broad patient populations into clinically relevant subgroups based on, for example, age [22], tumor biomarkers [23, 24], and primary tumor site [25]. Several of these factors, such as RAS mutation status and primary tumor site, now guide the use of epidermal growth factor receptor inhibitors, but there are as yet no established markers that inform treatment decisions for chemotherapy and bevacizumab. Furthermore, clinical trials are limited in the number of patient and disease characteristic combinations they can investigate, and combinations of a limited number of characteristics only partly explain the variation in clinical outcomes. Additionally, considering the success or failure of previous interventions in clinical trials for second and later lines of therapy adds to the required number of studies to be performed. Hence, it may not be possible to address challenges regarding treatment sequencing in $\mathrm{mCRC}$ by performing clinical trials only.

Observational studies based on real-world data (RWD) have been recognized as a valuable source of evidence for comparative effectiveness research in oncology, when evidence from clinical trials is not available or when results from clinical trials only apply to a selected group of patients $[26,27]$. In mCRC, for example, RWD have been used to describe observed treatment sequences, patient characteristics, and clinical outcomes [28], and to study the comparative effectiveness of FOLFOX and FOLFIRI sequencing (i.e., which chemotherapy doublet should be used in first-line and which in second-line treatment [29]). RWD are also being used to evaluate the prognostic role of inflammatory markers in patients treated with bevacizumab [30]. Although observational studies are subject to selection bias, careful analysis of RWD has the potential to provide valuable information on the comparative effectiveness of different treatment sequences.

Regardless of whether clinical outcomes of different treatment sequences for a patient population are studied through clinical trials or observational studies, it is challenging to translate results from these studies into an optimal sequencing strategy that informs treatment decisions across multiple lines of treatment. Simulation models developed based on individual patient data of patient and disease characteristics and treatment history have the potential to estimate the effectiveness, and impact, of different treatment combinations and sequencing strategies for future patient subpopulations. By applying such simulation models in combination with optimization techniques, optimal treatment sequencing strategies for specific patient subpopulations may be identified.

As an initial step towards modeling sequences across multiple treatment lines, this study aimed to use simulation modeling methods based on RWD to evaluate first-line mCRC treatment targeting with doublet chemotherapy with or without bevacizumab. A simulation model combining patient and disease characteristics and conditional treatment outcomes to estimate effectiveness of treatment sequencing strategies was developed based on data from the Treatment of Recurrent and Advanced Colorectal Cancer registry (TRACC) [31].

\section{Methods}

A discrete event simulation (DES; i.e., time-to-event simulation) was developed to simulate progression-free survival (PFS) and overall survival (OS) (Fig. 1). Time-to-events in the DES were simulated using two parametric survival models and one logistic regression-a survival model representing PFS for first-line treatment (Model L1-Time), a logistic regression to distinguish between the competing events of death during first-line treatment versus physician-defined disease progression (Model L1-Event), and a survival model representing OS for subsequent treatment lines or follow up after first-line progression (Model LX-Time). More details on how these models and the DES were developed and validated are provided in the following sections. 


\subsection{Treatment of Recurrent and Advanced Colorectal Cancer Registry (TRACC)}

TRACC was established to collect RWD about the treatments and outcomes of consecutive patients diagnosed with mCRC at multiple public and private hospitals across and outside Australia [31]. To date, almost 3000 patients have been enrolled in TRACC, and detailed patient and disease characteristics, treatment history of primary disease, and metastatic treatment motivation and outcomes across multiple treatment lines were all collected prospectively. TRACC data have been used previously to assess, for example, trends in biological agent use [32], primary tumor site impact on PFS and OS [33], and PFS, OS, and several safety endpoints for patients with an intact compared with a resected primary tumor [34].

\subsection{Data Cleaning and Categorization}

For every variable in TRACC, unique values and distributions were evaluated for data entry errors and for combinations of values with a similar meaning, as inconsistencies may have occurred following registry updates. As an example of a data entry error, a small number of patients had progression dates prior to treatment start dates, resulting in a negative PFS, and were excluded. Additionally, since patients typically receive different chemotherapy drugs in combination with a biological agent as part of first-line treatment, these different treatment combinations needed to be categorized to allow patient selection (see Sect. 2.3). Following a previously used categorization strategy, chemotherapy regimens were categorized according to the most intensive therapy, that is, singlet, doublet, or triplet chemotherapy (one, two, or three chemotherapy drugs given in combination), and the biological agent according to the first exposed biological [32].

\subsection{Patient Inclusion}

Patients treated in an Australian center, indicated for palliative-intent treatment, with a treatment start date after 1 January 2009 and before 1 June 2017, and with first-line treatment categorized as doublet chemotherapy with or without bevacizumab, were included for analysis. Physiciandefined treatment intent is captured in TRACC and was used to determine whether treatment was administered with curative (e.g., resectable liver metastases), potentially curative (may become resectable if an excellent response is achieved to initial chemotherapy), or palliative goals. The timeframe was chosen because bevacizumab was listed on the Australian Pharmaceutical Benefits Scheme in 2009 and to allow for a reasonable follow-up period to capture events.

\subsection{Variables Considered for Inclusion in the Models}

Candidate variables, transformations, and interactions for both parametric survival models were identified considering their clinical relevance and number of observations. The following variables were identified: age, gender, hospital type (private or public), stage at diagnosis, primary tumor site, presence of liver metastases, presence of peritoneal metastases, number of metastatic sites, Eastern Cooperative Oncology Group (ECOG) performance status, Charlson comorbidity index (not corrected for age), RAS mutation status (mutated or wild type), and BRAF mutation status. An interaction between the number of metastatic sites (1 or 2 vs $>2)$ and carcinoembryonic antigen (CEA) level $(<200$ vs $\geq 200$ ) was identified. A treatment indicator (chemotherapy and bevacizumab vs chemotherapy only) was included in all models. First-line PFS was included as a candidate variable for modeling OS after first-line progression (Model LX-Time) to make this survival model conditional on firstline treatment outcomes. An overview of all considered transformations is provided in Supplementary Materials 1 [see electronic supplementary material (ESM)]. For both survival models separately, each identified candidate variable and interaction was scored on its clinical relevance (i.e., low, moderate, or high) to guide variable selection in the multivariable analysis [see Sect. 2.7 and Supplementary Materials 2 (ESM)].

\subsection{Missing Data}

Data were missing for the following variables (\% missing): stage at initial diagnosis of colorectal cancer (1\%), primary tumor site (2\%), CEA level (9\%), RAS mutation

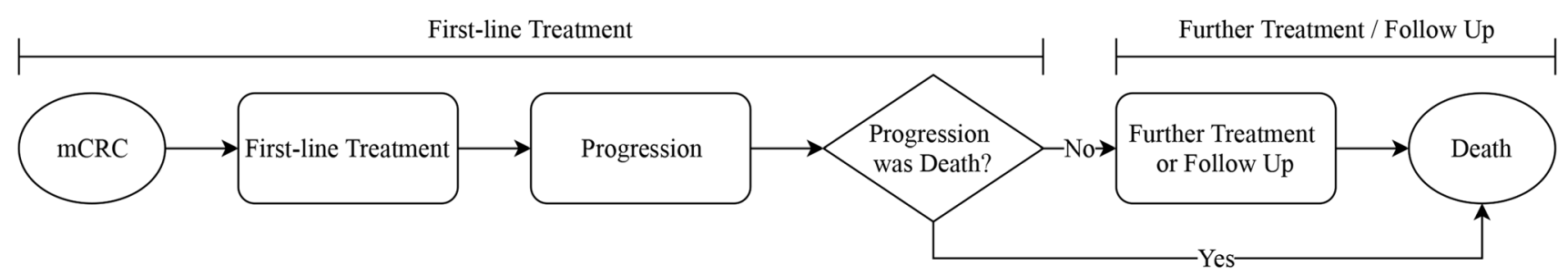

Fig. 1 Graphical representation of the discrete event simulation model structure 
status (20\%), and BRAF mutation status (57\%). The substantial number of observations missing for RAS and BRAF mutation status were due to this molecular testing not being routinely assessed throughout the selected timeframe. Missing data for RAS and BRAF mutation status were partly addressed by assuming that RAS and BRAF mutations are mutually exclusive [35]. Remaining missing data for RAS and BRAF mutation status and for other variables were imputed 25 times using multiple imputation with chained equations in 20 iterations [36, 37]. CEA level was imputed using Bayesian linear regression, RAS and BRAF mutation status were imputed using logistic regression, and stage at diagnosis and primary tumor site were imputed using multinomial logit models. All variables were used as predictors. Post-processing was performed to correct a positive BRAF mutation status to negative in the presence of a RAS mutation.

\subsection{Univariable Modeling}

Univariable modeling was performed to determine which distribution types would be used to parameterize the survival models and which transformations would be used for each variable in the multivariable modeling (see Sect. 2.7). First, a distribution type was selected for each survival model based on the likelihood and assessment of quantile-quantile plots and Kaplan-Meier plots. After considering exponential, Weibull, logistic, and log-normal distributions, a Weibull distribution was selected to parameterize each survival model (see Supplementary Materials 3 (ESM) for details). Next, best-performing transformations were selected for each survival model separately by fitting individual models for each candidate transformation and comparing performance based on Wald test $p$-values. Final selection of the transformation to be used was based on the average transformation rank over all 25 imputed datasets.

\subsection{Multivariable Modeling of Survival}

Two multivariable survival models Model L1-Time and Model LX-Time were developed to represent PFS for firstline treatment and OS following progression after first-line treatment accounting for subsequent treatment lines. To take both clinical relevance and statistical performance of variables into account, a comprehensive variable selection procedure based on variables' $p$-values was implemented for both survival models. Variables considered highly relevant were included regardless of their statistical performance, whereas variables with a moderate or low clinical relevance were included based on a backward or forward selection procedure based on their $p$-value, respectively. Treatment interactions were subsequently considered for all included variables and included based on a backward selection procedure (see Supplementary Materials 4 (ESM) for details). Clinical relevance was not considered for logistic regression Model L1-Event, as it is difficult to define variables' relevance in distinguishing between multiple competing events, and all candidate variables and their treatment interactions were included based on a forward selection procedure. To further value clinical relevance, relatively high $p$-value thresholds were chosen: 0.3 for all backward selections and 0.2 for all forward selections.

According to best practice for selection procedures using imputed data, an exhaustive approach was taken to calculate $p$-values for variables [38]. In each step of a selection procedure, a (survival or logistic regression) model was fitted to all imputed datasets and Rubin's rule [39] was applied to combine these 25 models into one model, including its total variance. Next, a Wald test $p$-value for each variable was calculated based on this pooled model, combining all coefficients that related to a specific variable. For example, variable Age was modeled using a second-order polynomial and, hence, inclusion or exclusion of Age as a variable was based on the two Age coefficients combined.

\subsection{Discrete Event Simulation (DES)}

A DES was populated using Model L1-Time, Model L1-Event, and Model LX-Time to simulate first-line PFS and OS on an individual patient level, reflecting patient heterogeneity and stochastic uncertainty (i.e., first-order uncertainty) (Fig. 1). To handle progression and death as competing events for first-line PFS, a unimodal joint distribution and regression model approach was implemented using Model L1-Time and Model L1-Event [40]. Although general guidance on implementing competing risks in DESs suggests using multimodal distributions, this guidance is for uncensored data [40]. Because our data had $6.5 \%$ censoring and time-to-event distributions overlapped substantially (73\%, Supplementary Materials 5, see ESM), it was decided to use a unimodal distribution. Simulations were performed for (subsets of) the TRACC patient population by randomly sampling 20,000 patients with replacement from the corresponding observed patient group.

\subsection{Probabilistic Analysis}

Probabilistic analysis was performed to reflect parameter uncertainty (i.e., second-order uncertainty). According to best practice for DES [41], correlated sets of model parameter coefficients were obtained by applying Rubin's rule [39] over all imputed datasets in each of 10,000 bootstrap iterations performed to assess internal validity (see Sect. 2.10 and Fig. 2). The resulting sets of Model L1-Time, Model L1-Event, and Model LX-Time coefficients were used to perform 10,000 probabilistic analysis runs. 


\subsection{Validation}

Several statistical measures were used to evaluate models' internal validity in terms of model discrimination (i.e., ability to distinguish between high- and low-risk individuals) and calibration (i.e., agreement between observed and predicted outcomes) [42]. Regarding survival model discrimination, Uno et al.'s [43] modification of Harrel's C-statistic [44] was used to account for censoring. Two measures were used to assess survival model calibration: (i) the regression slope of the predicted Weibull scale parameter in a Coxregression model [45], and (ii) Demler et al.'s GreenwoodNam-D'Agostino statistic (GND) [46], which is a modification of Hosmer-Lemeshow's statistic by D'Agostino and Nam [47]. Essentially, the modified C-statistic compares all possible patient pairs with respect to whether there is an agreement in the predicted risk in terms of whether it is higher or lower and their observed time-to-event or censoring time. In other words, it checks whether the time-to-event for a patient is indeed higher if predicted risk of the event is lower compared with another patient. The statistic is then calculated as the proportion of correct classifications; higher means better agreement. The GND assesses how well the survival probability of risk subgroups as predicted by the model (i.e., patients grouped based on the predicted probability of survival at a specific point in time) match the observed probability of survival for those patients in the data according to the Kaplan-Meier estimate. Both C-statistic and GND were estimated at discrete timepoints: $0.5,1$, and 2 years. Additionally, GND requires a number of groups to be specified in which patients are stratified according to their predicted risk, for which 5 and 10 were chosen to assess impact of this arbitrary decision. Internal validity of logistic regression Model L1-Event was assessed by excluding patients for whom no event was observed and using Harrel's C-statistic for model discrimination [44]. To assess calibration, the regression slope of the predicted response of Model L1-Event in a logistic regression model [48] and the Brier score [49] were used.

Following best practice [50], nearly unbiased estimates for all statistics were obtained by performing 10,000 bootstrap iterations (Fig. 2). In this process, the apparent performance of a model $D_{\text {app }}$, which is obtained by validating this model on all 25 imputed original datasets, is corrected for model optimism $\mathrm{O}$. This optimism $\mathrm{O}$ is calculated as the difference between the internal validity of a bootstrap model based on the bootstrap dataset $D_{\text {boot }}$ and the original dataset $D_{\text {origin }}[44,50]$.

To validate the DES, simulated median time-to-events and Kaplan-Meier plots (including confidence intervals (CIs) based on 10,000 probabilistic analysis runs, see Sect. 2.9) were compared with those observed.

\subsection{Reproducibility and Availability of Material}

All analyses and simulations were performed in $\mathrm{R}$ version 3.5.2 [51]. An online tool was made publicly available in which dummy TRACC data can be visualized and simulations using the DES can be performed. Additionally, R code written to perform all analyses and simulations was made available to enhance transparency and reproducibility. All these materials are available from https://personex.nl/resea rch/morc-tracc/.

\section{Results}

Of 2689 patients treated in an Australian hospital contributing data to the TRACC registry, 871 patients met the inclusion criteria. After excluding 4 of these patients due to negative PFS, data from 867 patients remained for analysis. Table 1 provides an overview of patient characteristics for patients treated with or without bevacizumab in addition to doublet chemotherapy (abbreviated as ChemBev and ChemOnly, respectively). There were more observations for the ChemBev group $(n=676)$ compared with the ChemOnly group $(n=191)$. Patients in the ChemBev group were significantly more likely to have received treatment in a private hospital, have a better ECOG performance status, and a lower Charlson co-morbidity index. Significant differences in RAS and BRAF status were found but are hard to interpret because of the large amount of missing data. RAS status was missing for 175 (20\%) patients and BRAF status was missing for 496 (57\%) patients. These numbers were reduced to 174 (20\%) and 354 (41\%), respectively, by assuming RAS and BRAF mutations to be mutually exclusive (see Sect. 2.5). Post-processing of BRAF mutation status following multiple imputation was only required in $0.35 \%$ of observations.

Model coefficients and 95\% CIs are presented in Supplementary Materials 2 (see ESM). For survival Model L1-Time, bias-corrected C-statistics were $0.65,0.62$, and 0.62 at $0.5,1$, and 2 years, respectively, indicating acceptable model discrimination. Regarding model calibration, a bias-corrected slope for the predicted Weibull Scale of 0.996 indicated good calibration. Bias-corrected GNDs ( $p$-values) of $8.7(0.07), 5.6(0.23)$, and $4.0(0.41)$ for five risk groups, and $18.6(0.03), 17.6(0.04)$, and $12.7(0.17)$ for ten risk groups, at $0.5,1$, and 2 years, respectively, indicated a good model calibration, except for short-term predictions (see Sect. 4 for a discussion). A graphical representation of uncorrected GNDs at 1 year is provided in Fig. 3, which shows predicted values compared with the observed values for one randomly selected imputed dataset. For logistic regression Model L1-Event, a bias-corrected C-statistic of 0.66 showed reasonable model discrimination. A biascorrected regression slope of 0.82 and Brier score of 0.09 
Fig. 2 Overview of the complete imputation and validation process. PSA probabilistic sensitivity analysis; *whether $O_{\text {mean }}$ is subtracted from or added to $D_{\text {app }}$ depends on the performance measure

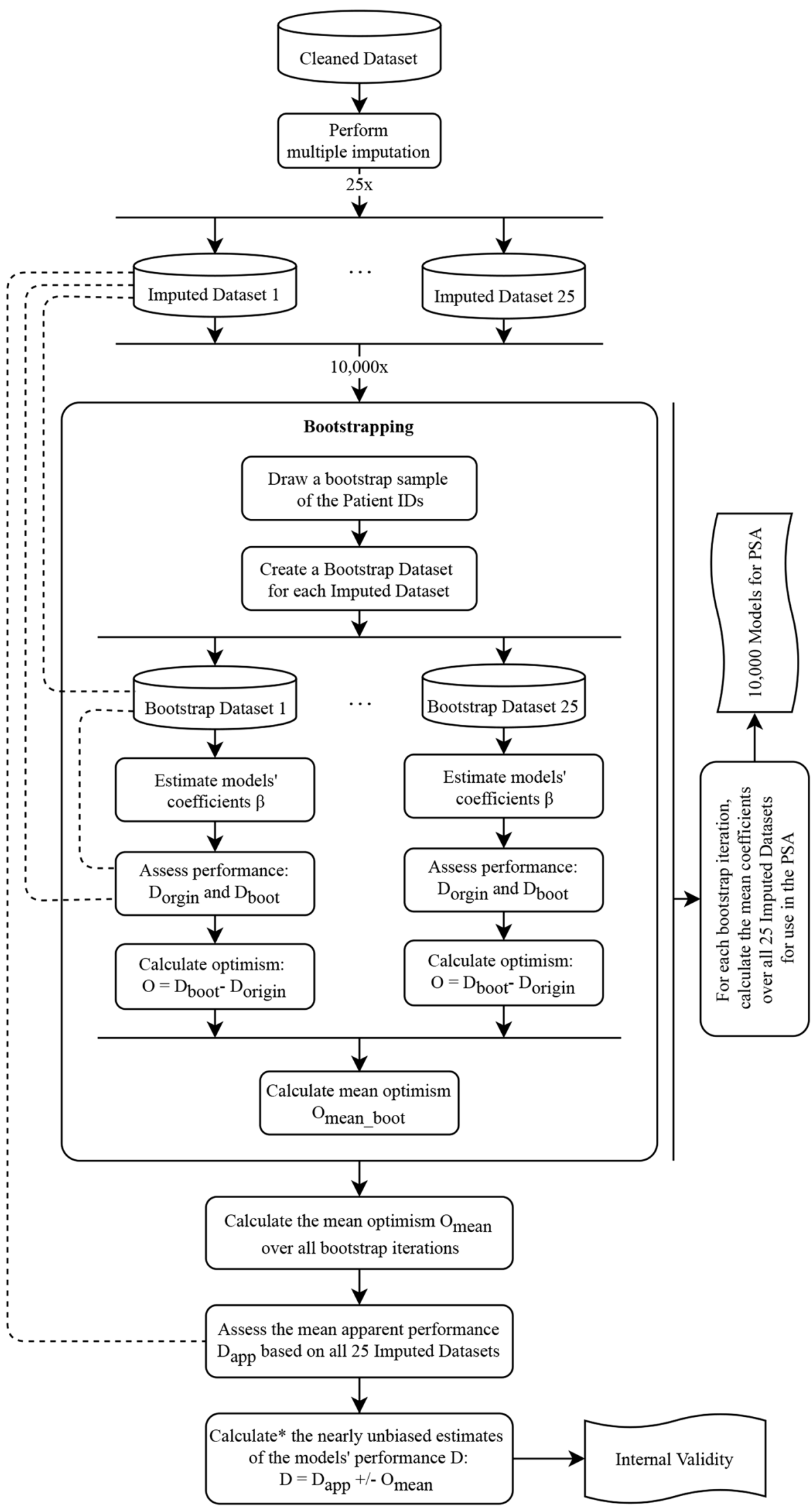


showed good model calibration. Bias-corrected C-statistics for survival Model LX-Time of 0.70, 0.68, and 0.67 at 0.5, 1 , and 2 years, respectively, indicated a reasonable model discrimination, and a bias-corrected slope for the predicted Weibull Scale of 0.998 indicated good calibration. Biascorrected GNDs and $p$-values of $12.0(0.02), 1.1(0.90)$, and $5.6(0.23)$ for five risk groups, and $26.9(<0.01), 11.3(0.26)$, and $13.3(0.15)$ for ten risk groups, at $0.5,1$, and 2 years, respectively, indicated good calibration, except for shortterm predictions (also see Fig. 3).

Simulated Kaplan-Meier plots were similar to those observed (Fig. 4). For first-line treatment, the simulated median days until progression (95\% CIs) were 199 (171-228) and 308 (289-328), and observed medians were $171(155-210)$ and 304 (280-329), for ChemOnly and ChemBev, respectively (Fig. 4a). Simulations yielded a median of 1171 days (1013-1392) for time until death during first-line treatment for ChemBev, and the observed median was 1055 days (Fig. 4b). No observed confidence intervals for ChemBev nor any information for ChemOnly regarding this time-to-event could be estimated due to a low number of events. Regarding OS after first-line treatment, simulated median days were 200 (161-242) and 266 (243-292), and observed medians were 193 (153-243) and 270 (237-308) for ChemOnly and ChemBev, respectively (Fig. 4c). In the simulation, 11.6\% (7.1-16.6) of patients died on first-line treatment in the ChemOnly group, whereas death was observed as event for $11.0 \%$ of the patients in the data, and for $6.8 \%$ of patients no event was observed. For ChemBev, $10.3 \%$ (7.9-12.9) of deaths were simulated, and death was observed as event for $9.2 \%$ of patients in the data, with $6.4 \%$ of patients being censored.

Exploratory scenario analyses using the DES estimated the impact of selecting the treatment most likely to be effective in terms of PFS on an individual level. These analyses estimated that median first-line PFS (95\% CI) of 219 (25\%) patients increased from 175 days (156-199) to 269 days (246-294) if a different treatment was selected (Fig. 5). On a population level, this resulted in an estimated improved median first-line PFS of 288 days (270-307) compared with 265 days (248-280) as observed from the data. For $168(77 \%)$ of these patients, ChemBev treatment was targeted rather than ChemOnly (observed), whereas ChemOnly rather than ChemBev (observed) was targeted for 51 (23\%) patients. As expected, based on the model coefficients (Supplementary Materials 2, see ESM), patients for whom ChemOnly was targeted rather than ChemBev typically had an ECOG performance score of 2 or more $(53 \%)$ or a CEA level of 200 or more and one or two metastatic sites (51\%). All these patients had also been treated in a private hospital. Most of the patients for whom ChemBev was targeted rather than ChemOnly had an ECOG performance score of 0 or 1 $(86 \%)$.
Table 1 Patient characteristics for the doublet chemotherapy only (ChemOnly) and doublet chemotherapy with bevacizumab (Chem$\mathrm{Bev}$ ) treatment strategies

\begin{tabular}{|c|c|c|c|}
\hline & ChemOnly & ChemBev & $p$ value \\
\hline Number of patients & 191 & 676 & \\
\hline Age & $63(14)$ & $63(12)$ & 0.571 \\
\hline \multicolumn{4}{|l|}{ Gender } \\
\hline Male & $118(62)$ & $407(60)$ & \multirow[t]{2}{*}{0.739} \\
\hline Female & $73(38)$ & $269(40)$ & \\
\hline \multicolumn{4}{|l|}{ Hospital type } \\
\hline Public & $115(60)$ & $337(50)$ & \multirow[t]{2}{*}{$0.014 * *$} \\
\hline Private & $76(40)$ & $339(50)$ & \\
\hline \multicolumn{4}{|l|}{ Stage at diagnosis } \\
\hline Locally advanced & $8(4)$ & $35(5)$ & \multirow[t]{6}{*}{0.294} \\
\hline Stage I & $0(0)$ & $11(2)$ & \\
\hline Stage II & $10(5)$ & $35(5)$ & \\
\hline Stage III & $21(11)$ & $95(14)$ & \\
\hline Stage IV & $151(79)$ & $491(73)$ & \\
\hline Missing & $1(1)$ & $9(1)$ & \\
\hline \multicolumn{4}{|l|}{ Primary tumor site } \\
\hline Left colon & $73(38)$ & $261(39)$ & \multirow[t]{5}{*}{0.361} \\
\hline Right colon & $51(27)$ & $213(32)$ & \\
\hline Colon NOS & $13(7)$ & $26(4)$ & \\
\hline Rectum & $51(27)$ & $165(24)$ & \\
\hline Missing & $3(2)$ & $11(2)$ & \\
\hline \multicolumn{4}{|c|}{ Number of metastatic sites } \\
\hline 1 & $91(48)$ & $256(38)$ & \multirow[t]{6}{*}{0.168} \\
\hline 2 & $55(29)$ & $254(38)$ & \\
\hline 3 & $33(17)$ & $123(18)$ & \\
\hline 4 & $9(5)$ & $36(5)$ & \\
\hline 5 & $3(2)$ & $6(1)$ & \\
\hline 6 & $0(0)$ & $1(0)$ & \\
\hline \multicolumn{4}{|l|}{ Liver metastases } \\
\hline Yes & $127(66)$ & $465(69)$ & \multirow[t]{2}{*}{0.606} \\
\hline No & $64(34)$ & $211(31)$ & \\
\hline \multicolumn{4}{|c|}{ Peritoneum metastases } \\
\hline Yes & $53(28)$ & $185(27)$ & \multirow[t]{2}{*}{0.926} \\
\hline No & $138(72)$ & $491(73)$ & \\
\hline \multicolumn{4}{|c|}{ ECOG performance status } \\
\hline 0 & $51(27)$ & $284(42)$ & \multirow[t]{4}{*}{$<0.001 * * *$} \\
\hline 1 & $95(50)$ & $344(51)$ & \\
\hline 2 & $34(18)$ & $43(6)$ & \\
\hline 3 & $11(6)$ & $5(1)$ & \\
\hline \multicolumn{4}{|c|}{ Charlson Comorbidity Index (age-unadjusted) } \\
\hline 0 & $127(66)$ & $487(72)$ & \multirow[t]{5}{*}{$0.001 * * *$} \\
\hline 1 & $27(14)$ & $125(18)$ & \\
\hline 2 & $22(12)$ & $41(6)$ & \\
\hline 3 & $7(4)$ & $18(3)$ & \\
\hline$\geq 4$ & $8(4)$ & $5(1)$ & \\
\hline \multicolumn{4}{|l|}{ CEA level } \\
\hline Recorded & $726(5254)$ & 478 (2049) & \multirow[t]{2}{*}{0.546} \\
\hline Missing & $19(10)$ & $61(9)$ & \\
\hline
\end{tabular}


Table 1 (continued)

\begin{tabular}{llll}
\hline & ChemOnly & ChemBev & $p$ value \\
\hline RAS status & & & \\
Mutated & $56(29)$ & $247(37)$ & $<0.001^{* * * *}$ \\
Wild-type & $72(38)$ & $317(47)$ & \\
$\quad$ Missing & $63(33)$ & $112(17)$ & \\
BRAF status & & & \\
Mutated & $12(6)$ & $41(6)$ & $0.006^{* * *}$ \\
Wild-type & $52(27)$ & $266(39)$ & \\
Missing & $127(66)$ & $369(55)$ & \\
\hline
\end{tabular}

Values represent the count (\%) for categorical variables and mean (SD) for continuous variables

CEA carcinoembryonic antigen, ECOG Eastern Cooperative Oncology Group, NOS not otherwise specified

Significance levels: $*<0.10 ; * *<0.05 ; * * *<0.01$

Simulations for specific subgroups of patients can be performed using the DES through the online tool that is available at: https://personex.nl/research/mcrc-tracc/.

\section{Discussion}

To our knowledge, this is the first mCRC simulation model that reflects patient heterogeneity based on RWD, combining patient and disease characteristics and conditional treatment outcomes, which can be used to estimate the effectiveness of different treatment combinations for specific patient subpopulations. Incorporation of RWD into simulation techniques has the potential to identify optimal treatment sequencing strategies for specific patient subpopulations, which is unlikely to ever be achieved by performing clinical trials because of an infeasible number of studies required to test all sequences and all patient subsets. Although simulations have been used in mCRC to estimate the cost effectiveness of, for example, bevacizumab in multiple countries [52], several common treatment sequences [53,54], and KRAS testing to target cetuximab treatment [55], these simulation studies did not account for patient heterogeneity and, therefore, cannot be used to identify optimal treatment strategies. Furthermore, RWD has been used previously to evaluate the
Fig. 3 Illustration of the Greenwood-Nam-D'Agostino statistic, showing predicted vs observed risks for $\mathbf{a}, \mathbf{b}$ first-line progression (Model L1-Time) and $\mathbf{c}, \mathbf{d}$ overall survival after progression (Model LX-Time), and the impact of selecting $\mathbf{a}, \mathbf{c}$ 5 risk groups and $\mathbf{b}, \mathbf{d} 10$ risk groups, at a 1-year timepoint and for one of the imputed datasets

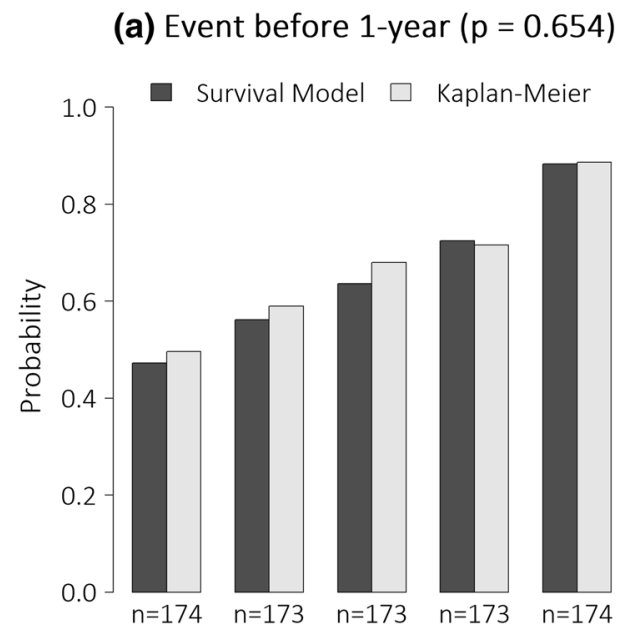

(c) Event before 1-year $(p=0.583)$

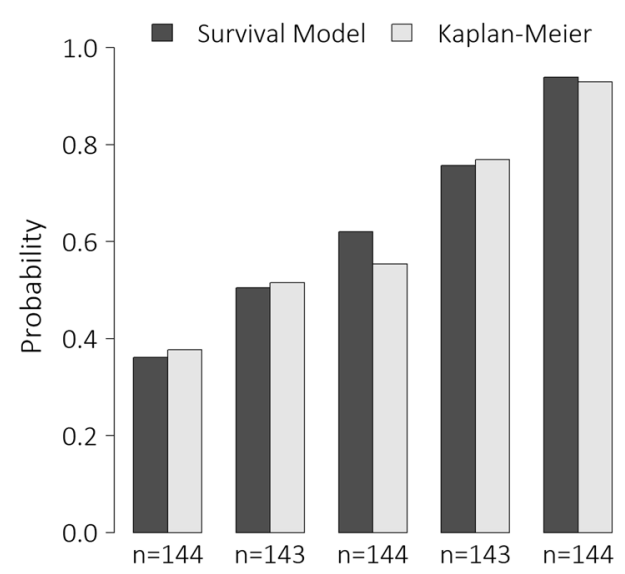

(b) Event before 1-year $(p=0.52)$

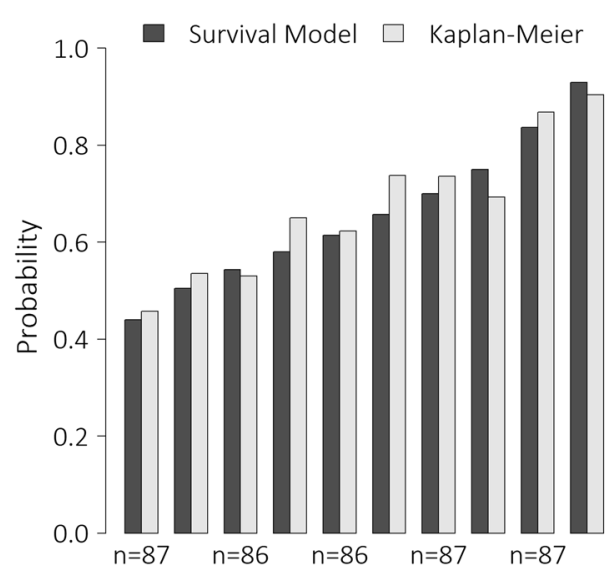

(d) Event before 1-year $(p=0.894)$

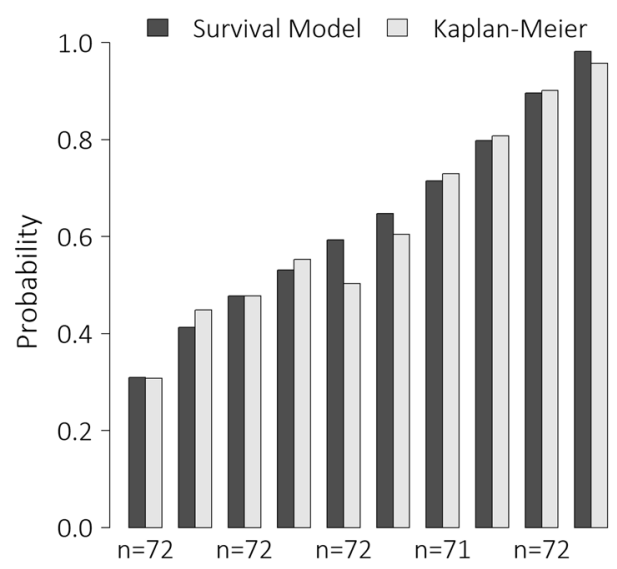


(a) First-Line Progression (Progression) ChemOnly: $n=157$, ChemBev: $n=571$

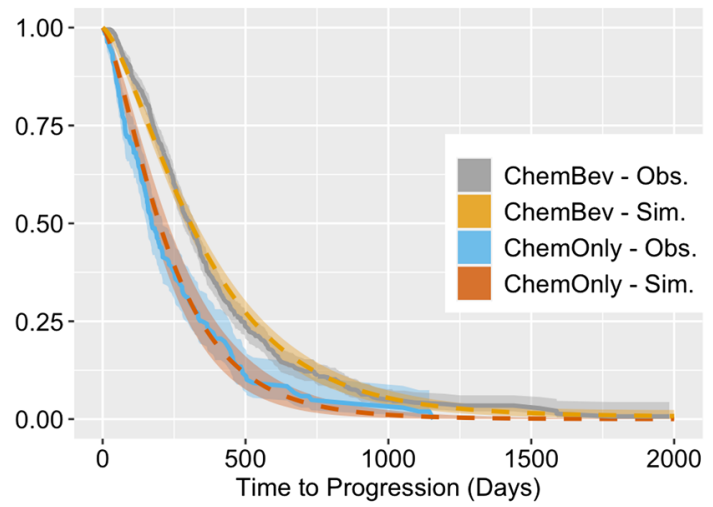

(b) First-Line Progression (Death)

ChemOnly: $n=21$, ChemBev: $n=62$

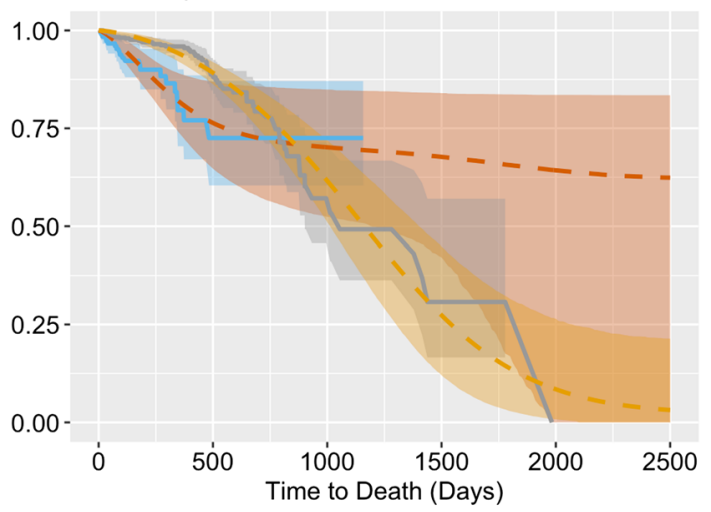

(c) Death after Progression

ChemOnly: $n=141$, ChemBev: $n=467$

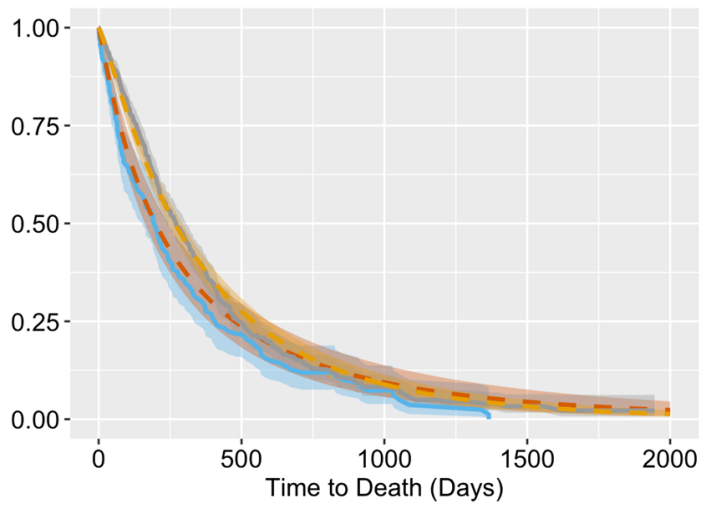

Fig. 4 Observed vs simulated Kaplan-Meier plots for the discrete event simulation model, including the number of events in the corresponding patient groups and 95\% confidence intervals (colored surface) based on the Kaplan-Meier estimate (observed) and based on the probabilistic sensitivity analysis (simulated), for a time-to-progression for patients who progress on first-line treatment, b time-todeath for patients who die on first-line treatment, and $\mathbf{c}$ time-to-death after progression for patients who progress on first-line treatment

overall outcome of different treatment sequences for mCRC in general [29], but as this study did not employ simulation modeling methods, no alternative treatment strategies, compared with what is in the registry, could be considered.

The multivariable survival models presented in this manuscript are versatile as they can be utilized in future studies to model progression-free and overall survival for first-line treatment of mCRC using doublet chemotherapy with or without bevacizumab. For example, the survival models can be used beyond DES to derive patient-level transition probabilities in state-transition models reflecting heterogeneity. When using results from this study, modelers should understand and acknowledge the limitations to our study as we describe below, as well as the characteristics of the patient population from which they were derived.

Given that a recent methodological review highlighted the need for improved reporting on validation of health economic models for mCRC [56], we performed and reported extensive validation efforts required to assess the suitability of the DES to inform decision making. Although the internal validation showed reasonable to good performance, these statistics are hard to interpret and subject to several arbitrary decisions. For example, C-statistics for survival models do not take into account time differences between individuals, which implies that classifying a patient that progressed at day 100 to be at higher risk than a patient that progressed at day 95 is as incorrect as classifying a patient that progressed at day 500 as at higher risk than a patient that progressed at day 40 . Hence, a discrimination statistic that considers the distance between individuals would be preferred, though we are not aware of such a statistic. Another aspect that should be considered when interpreting statistics such as the C-statistic and GND for survival models is the timepoints at which these are evaluated. For the GND specifically, the selected number of risk groups may also impact model performance outcomes. Furthermore, depending on the intended use of survival models, either model discrimination or calibration may be considered more important. Here, the aim is to predict different time-to-events for an individual and model calibration may be considered most relevant. Finally, it is unknown how model performance is influenced by modeling the time-to-event of two competing events (i.e. progression and death) using one parametric survival model.

This study shows that there is potential for translational research using RWD and simulation techniques; however, it is subject to limitations associated with using this source of evidence. First, only 191 patients were included for the ChemOnly treatment strategy compared with 676 patients for the ChemBev strategy, whereas clinical trials would have typically yielded groups of similar sizes. The impact of the relatively low sample size for the ChemOnly strategy is illustrated in Fig. 4b, which shows that the low number of deaths seen in ChemOnly patients results in a markedly different-looking curve compared with the ChemBev strategy. 
Fig. 5 Kaplan-Meier plots illustrating the progression-free survival (solid lines) and 95\% confidence intervals (dashed lines) for the personalized treatment strategy compared with treatment as observed in the data, for a the patients that switch treatment according to the personalized treatment strategy and $\mathbf{b}$ the complete patient population
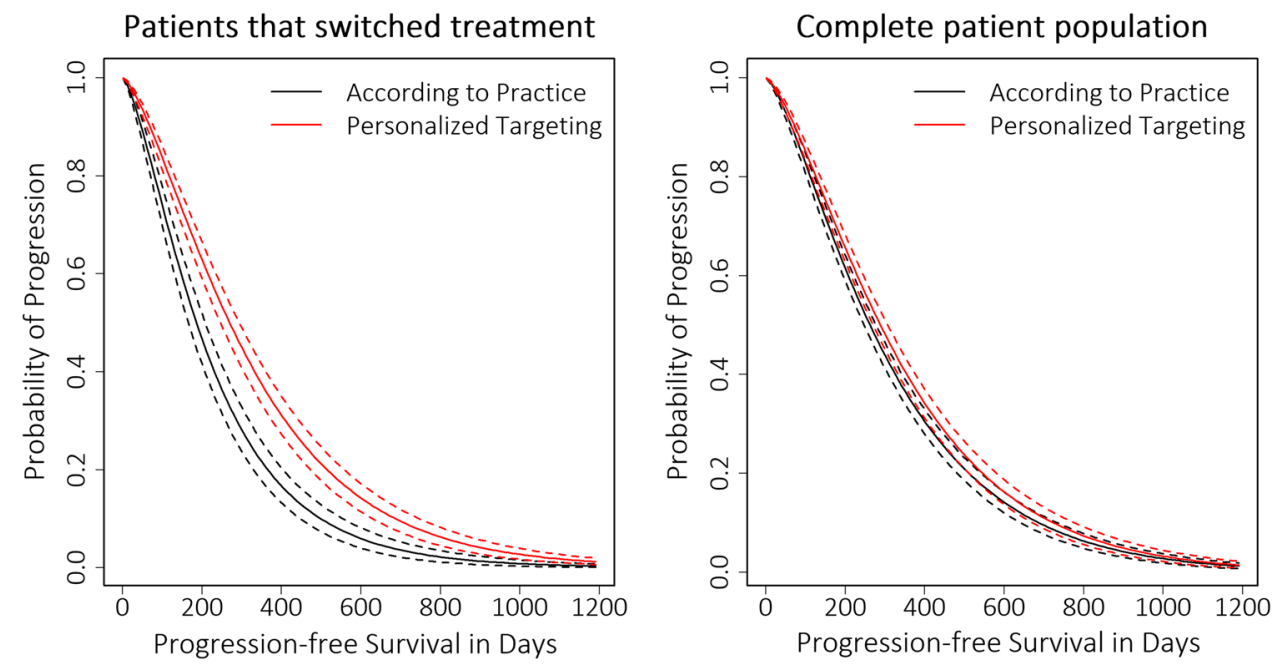

Additionally, significant differences were observed in important prognostic patient characteristics, such as ECOG performance status, highlighting the risk of selection bias as one of the potential drivers of differences in survival outcomes. Although only patients with a palliative treatment intent were included and multivariable models were developed, it cannot be assumed that the possibility of selection bias in the treatment effect coefficients has been fully eliminated. Use of propensity score weighting or matching to address selection bias could be investigated, however this may result in increased uncertainty surrounding simulation outcomes given the low number of patients for the ChemOnly strategy and expected loss of (effective) sample size.

The study methods have certain limitations. A different treatment categorization strategy could have been applied, which might have resulted in inclusion of a different patient population. Also, patients could have been restricted to those treated at a later moment in time to reduce the proportion of missing values for the RAS and BRAF mutational status. However, this would have decreased the number of observations substantially, being particularly troublesome for the ChemOnly treatment strategy. We excluded patients treated with first-line chemotherapy plus an epidermal growth factor receptor inhibitor due to small numbers, but this strategy needs to be considered in future modeling as uptake is increasing, particularly among patients with left-sided RAS wild-type tumors [32]. Finally, a rather inclusive variable selection procedure with high $p$-value thresholds was implemented to value clinical relevance. Although other thresholds, procedures, and criteria, such as using the Akaike information criterion, could have been used, it is unknown whether this would have resulted in different models.

Despite challenges regarding the selection and interpretation of validation measures, an important question is when models are to be considered 'good enough'; that is, when are simulations accurate enough to be useful? Although no minimum level of performance was defined beforehand, performance was considered sufficient to estimate population-level outcomes. Therefore, the simulation model can be used to assess the impact of different treatment decisions at the population level; for example, to evaluate observed differences between treatments as observed from the data and clinical practice according to the clinical guidelines. A next step in order to increase the informative value of the DES would be to add further first-line treatment outcomes, such as best-achieved response and quality of life, as well as second-line treatment and outcomes. Additionally, treatment costs and costs of treating adverse events may be added to provide information on the cost effectiveness of different treatment sequencing strategies. Using such an approach to simulation of health economic outcomes, optimization algorithms can be applied to obtain insights into key costeffectiveness drivers and, ultimately, identify a personalized sequencing strategy that optimizes clinical outcomes given certain resource constraints. Although such personalized simulations have great potential for providing information on challenging treatment sequencing questions, they also pose questions regarding how complex these simulations can get before stakeholders no longer understand (and believe) them.

Based on the performance of multivariable survival models and general limitations of using RWD, the model presented in this manuscript aims to estimate population-level outcomes; however, it is important to note that this simulation modeling approach is not yet appropriate to inform treatment decisions at the individual patient level as this requires further external validation. While the estimation of survival outcomes of treatment combinations was the main objective of this study, a second goal was to demonstrate and advance the use of RWD to develop patient-level models in general. For instance, we developed and used $\mathrm{R}$ code for variable selection for parametric survival distributions based on multiple imputed datasets. This code has been made 
available with this manuscript to help future work in this space, as missing data are common in RWD. Additionally, we developed $\mathrm{R}$ code and demonstrated performance measures for validating multivariable survival models, which is useful for future health economic modeling studies.

\section{Conclusions}

This study has demonstrated and explored the development and validation of a DES utilizing RWD to estimate PFS and OS for different first-line treatment combinations for specific mCRC patient subgroups. Importantly, we found that targeting bevacizumab for specific mCRC subgroups can potentially increase PFS in our real-world patient cohort. These findings show that simulation models utilizing RWD have potential to optimize treatment sequencing strategies for specific patient subpopulations when data from randomized studies are lacking. However, suitability of simulations for this purpose should be assessed by thorough and transparent validation. Researchers are encouraged to utilize the multivariable survival models and $\mathrm{R}$ code that have been made publicly available with this manuscript, whereas others may explore the online tool that allows for data exploration and simulations using the DES.

Author contributions KD, HLW, HK, PG, and MIJ contributed to the study conception and design. KD analyzed the data in close collaboration with HLW and under supervision of PG and MIJ. KD developed the simulation under supervision of HK and MIJ. All authors contributed to the interpretation and discussion of the results. The first draft of the manuscript was prepared by KD and HLW, and critically reviewed by all other authors. All authors read and approved the final manuscript.

Data and code availability statement All R code, including intermediate and final outcomes, as well as a tool that allows exploration of dummy data similar to the TRACC data and simulations using the DES to be performed, are available online at https://personex.nl/research/ mcrc-tracc/. Data from the TRACC registry is not publicly available.

\section{Compliance with Ethical Standards}

Funding This research was partly funded by the Netherlands Organisation for Health Research and Development (ZonMW) as part of the Translational Research Program (project number: 446001006). Roche Products Pty Limited (Australia) provided financial assistance for the development, installation and maintenance of the TRACC database. BioGrid Australia manages the TRACC database and provided data access and support.

Conflict of interest MB has served on an advisory board to Roche, the manufacturer of bevacizumab. The authors declare that there is no conflict of interest regarding the publication of this article.

\section{References}

1. Van Cutsem E, Cervantes A, Nordlinger B, Arnold D. Metastatic colorectal cancer: ESMO Clinical Practice Guidelines for diagnosis, treatment and follow-up. Ann Oncol. 2014;25(Suppl 3):1-9.

2. Douillard JY, Cunningham D, Roth AD, Navarro M, James RD, Karasek P, et al. Irinotecan combined with fluorouracil compared with fluorouracil alone as first-line treatment for metastatic colorectal cancer: a multicentre randomised trial. Lancet. 2000;355(9209):1041-7.

3. Saltz LB, Cox JV, Blanke C, Rosen LS, Fehrenbacher L, Moore $\mathrm{MJ}$, et al. Irinotecan plus fluorouracil and leucovorin for metastatic colorectal cancer. Irinotecan Study Group. N Engl J Med. 2000;343(13):905-14.

4. de Gramont A, Figer A, Seymour M, Homerin M, Hmissi A, Cassidy J, et al. Leucovorin and fluorouracil with or without oxaliplatin as first-line treatment in advanced colorectal cancer. J Clin Oncol. 2000;18(16):2938-47.

5. Giacchetti S, Perpoint B, Zidani R, Le Bail N, Faggiuolo R, Focan C, et al. Phase III multicenter randomized trial of oxaliplatin added to chronomodulated fluorouracil-leucovorin as first-line treatment of metastatic colorectal cancer. J Clin Oncol. 2000;18(1):136-47.

6. Kohne CH, van Cutsem E, Wils J, Bokemeyer C, El-Serafi M, Lutz MP, et al. Phase III study of weekly high-dose infusional fluorouracil plus folinic acid with or without irinotecan in patients with metastatic colorectal cancer: European Organisation for Research and Treatment of Cancer Gastrointestinal Group Study 40986. J Clin Oncol. 2005;23(22):4856-65.

7. Hurwitz H, Fehrenbacher L, Novotny W, Cartwright T, Hainsworth J, Heim W, et al. Bevacizumab plus irinotecan, fluorouracil, and leucovorin for metastatic colorectal cancer. N Engl J Med. 2004;350(23):2335-42.

8. Cunningham D, Lang I, Marcuello E, Lorusso V, Ocvirk J, Shin $\mathrm{DB}$, et al. Bevacizumab plus capecitabine versus capecitabine alone in elderly patients with previously untreated metastatic colorectal cancer (AVEX): an open-label, randomised phase 3 trial. Lancet Oncol. 2013;14(11):1077-85.

9. Guan ZZ, Xu JM, Luo RC, Feng FY, Wang LW, Shen L, et al. Efficacy and safety of bevacizumab plus chemotherapy in Chinese patients with metastatic colorectal cancer: a randomized phase III ARTIST trial. Chin J Cancer. 2011;30(10):682-9.

10. Kabbinavar FF, Schulz J, McCleod M, Patel T, Hamm JT, Hecht $\mathrm{JR}$, et al. Addition of bevacizumab to bolus fluorouracil and leucovorin in first-line metastatic colorectal cancer: results of a randomized phase II trial. J Clin Oncol. 2005;23(16):3697-705.

11. Tebbutt NC, Wilson K, Gebski VJ, Cummins MM, Zannino D, van Hazel GA, et al. Capecitabine, bevacizumab, and mitomycin in first-line treatment of metastatic colorectal cancer: results of the australasian gastrointestinal trials group randomized phase III MAX study. J Clin Oncol. 2010;28(19):3191-8.

12. Goldberg RM, Sargent DJ, Morton RF, Fuchs CS, Ramanathan RK, Williamson SK, et al. A randomized controlled trial of fluorouracil plus leucovorin, irinotecan, and oxaliplatin combinations in patients with previously untreated metastatic colorectal cancer. J Clin Oncol. 2004;22(1):23-30.

13. Tournigand C, Andre T, Achille E, Lledo G, Flesh M, MeryMignard D, et al. FOLFIRI followed by FOLFOX6 or the reverse sequence in advanced colorectal cancer: a randomized GERCOR study. J Clin Oncol. 2004;22(2):229-37.

14. Falcone A, Ricci S, Brunetti I, Pfanner E, Allegrini G, Barbara C, et al. Phase III trial of infusional fluorouracil, leucovorin, oxaliplatin, and irinotecan (FOLFOXIRI) compared with infusional fluorouracil, leucovorin, and irinotecan (FOLFIRI) as first-line 
treatment for metastatic colorectal cancer: the Gruppo Oncologico Nord Ovest. J Clin Oncol. 2007;25(13):1670-6.

15. Passardi A, Nanni O, Tassinari D, Turci D, Cavanna L, Fontana A, et al. Effectiveness of bevacizumab added to standard chemotherapy in metastatic colorectal cancer: final results for first-line treatment from the ITACa randomized clinical trial. Ann Oncol. 2015;26(6):1201-7.

16. Saltz LB, Clarke S, Díaz-Rubio E, Scheithauer W, Figer A, Wong $\mathrm{R}$, et al. Bevacizumab in combination with oxaliplatin-based chemotherapy as first-line therapy in metastatic colorectal cancer: a randomized phase III study. J Clin Oncol. 2008;26(12):2013-9.

17. Cremolini C, Loupakis F, Antoniotti C, Lupi C, Sensi E, Lonardi $\mathrm{S}$, et al. FOLFOXIRI plus bevacizumab versus FOLFIRI plus bevacizumab as first-line treatment of patients with metastatic colorectal cancer: updated overall survival and molecular subgroup analyses of the open-label, phase 3 TRIBE study. Lancet Oncol. 2015;16(13):1306-15.

18. Loupakis F, Cremolini C, Masi G, Lonardi S, Zagonel V, Salvatore $\mathrm{L}$, et al. Initial therapy with FOLFOXIRI and bevacizumab for metastatic colorectal cancer. N Engl J Med. 2014;371(17):1609-18.

19. Simkens LHJ, van Tinteren H, May A, ten Tije AJ, Creemers GJM, Loosveld OJL, et al. Maintenance treatment with capecitabine and bevacizumab in metastatic colorectal cancer (CAIRO3): a phase 3 randomised controlled trial of the Dutch Colorectal Cancer Group. Lancet. 2015;385(9980):1843-52.

20. Loree JM, Kopetz S. Recent developments in the treatment of metastatic colorectal cancer. Ther Adv Med Oncol. 2017;9(8):551-64.

21. Temraz S, Mukherji D, Shamseddine A. Sequencing of treatment in metastatic colorectal cancer: where to fit the target. World J Gastroenterol. 2014;20(8):1993-2004

22. Aparicio T, Desrame J, Lecomte T, Mitry E, Belloc J, Etienney I, et al. Oxaliplatin- or irinotecan-based chemotherapy for metastatic colorectal cancer in the elderly. Br J Cancer. 2003;89(8):1439-44.

23. Stintzing S, Modest DP, Rossius L, Lerch MM, von Weikersthal LF, Decker T, et al. FOLFIRI plus cetuximab versus FOLFIRI plus bevacizumab for metastatic colorectal cancer (FIRE-3): a post-hoc analysis of tumour dynamics in the final RAS wild-type subgroup of this randomised open-label phase 3 trial. Lancet Oncol. 2016;17(10):1426-34.

24. Loupakis F, Cremolini C, Salvatore L, Masi G, Sensi E, Schirripa $\mathrm{M}$, et al. FOLFOXIRI plus bevacizumab as first-line treatment in BRAF mutant metastatic colorectal cancer. Eur J Cancer. 2014;50(1):57-63.

25. Arnold D, Lueza B, Douillard JY, Peeters M, Lenz HJ, Venook A, et al. Prognostic and predictive value of primary tumour side in patients with RAS wild-type metastatic colorectal cancer treated with chemotherapy and EGFR directed antibodies in six randomized trials. Ann Oncol. 2017;28(8):1713-29.

26. Giordano SH. Comparative effectiveness research in cancer with observational data. American Society of Clinical Oncology Educational Book. 2015;35:e330-5.

27. Hershman DL, Wright JD. Comparative effectiveness research in oncology methodology: observational data. J Clin Oncol. 2012;30(34):4215-22

28. McLean J, Rho YS, Kuruba G, Mamo A, Gilabert M, Kavan T, et al. Clinical practice patterns in chemotherapeutic treatment regimens for metastatic colorectal cancer. Clin Colorectal Cancer. 2016;15(2):135-40.

29. Teng C-LJ, Wang C-Y, Chen Y-H, Lin C-H, Hwang W-L. Optimal sequence of irinotecan and oxaliplatin-based regimens in metastatic colorectal cancer: a population-based observational study. PLoS One. 2015;10(8):e0135673.

30. Clarke S, Burge M, Cordwell C, Gibbs P, Reece W, Tebbutt N. An Australian translational study to evaluate the prognostic role of inflammatory markers in patients with metastatic ColorEctal
caNcer Treated with bevacizumab (Avastin) [ASCENT]. BMC Cancer. 2013;13:120.

31. Field K, Wong H-L, Shapiro J, Kosmider S, Tie J, Bae S, et al. Developing a national database for metastatic colorectal cancer management: perspectives and challenges. Intern Med J. 2013;43(11):1224-31.

32. Semira C, Wong H-L, Field K, Lee M, Lee B, Nott L, et al. Chemotherapy and biologic use in the routine management of metastatic colorectal cancer in Australia: is clinical practice following the evidence? Intern Med J. 2019;49(4):446-54.

33. Wong H-L, Lee B, Field K, Lomax A, Tacey M, Shapiro $\mathrm{J}$, et al. Impact of primary tumor site on bevacizumab efficacy in metastatic colorectal cancer. Clin Colorectal Cancer. 2016;15(2):e9-15.

34. Lee B, Wong H-L, Tacey M, Tie J, Wong R, Lee M, et al. The impact of bevacizumab in metastatic colorectal cancer with an intact primary tumor: results from a large prospective cohort study. Asia Pac J Clin Oncol. 2017;13(4):314-21.

35. Rajagopalan H, Bardelli A, Lengauer C, Kinzler KW, Vogelstein $\mathrm{B}$, Velculescu VE. Tumorigenesis: RAF/RAS oncogenes and mismatch-repair status. Nature. 2002;418(6901):934.

36. Van Buuren S, Brand JPL, Groothuis-Oudshoorn CGM, Rubin DB. Fully conditional specification in multivariate imputation. J Stat Comput Simul. 2006;76(12):1049-64.

37. Van Buuren S, Groothuis-Oudshoorn K. mice: multivariate imputation by chained equations in R. J Stat Softw. 2011;45(3):1-67.

38. Wood AM, White IR, Royston P. How should variable selection be performed with multiply imputed data? Stat Med. 2008;27(17):3227-46.

39. Rubin DB, Schenker N. Multiple imputation for interval estimation from simple random samples with ignorable nonresponse. J Am Stat Assoc. 1986;81(394):366-74.

40. Degeling K, Koffijberg H, Franken MD, Koopman M, IJzerman MJ. Comparing strategies for modeling competing risks in discrete-event simulations: a simulation study and illustration in colorectal cancer. Med Decis Making. 2019;39(1):57-73.

41. Degeling K, IJzerman MJ, Koopman M, Koffijberg H. Accounting for parameter uncertainty in the definition of parametric distributions used to describe individual patient variation in health economic models. BMC Med Res Methodol. 2017;17(1):170.

42. Rahman MS, Ambler G, Choodari-Oskooei B, Omar RZ. Review and evaluation of performance measures for survival prediction models in external validation settings. BMC Med Res Methodol. 2017; 17(1):60.

43. Uno H, Cai T, Pencina MJ, D’Agostino RB, Wei LJ. On the $\mathrm{C}$-statistics for evaluating overall adequacy of risk prediction procedures with censored survival data. Stat Med. 2011;30(10):1105-17.

44. Harrell FE Jr, Lee KL, Mark DB. Multivariable prognostic models: issues in developing models, evaluating assumptions and adequacy, and measuring and reducing errors. Stat Med. 1996;15(4):361-87.

45. Van Houwelingen HC. Validation, calibration, revision and combination of prognostic survival models. Stat Med. 2000;19(24):3401-15.

46. Demler OV, Paynter NP, Cook NR. Tests of calibration and goodness-of-fit in the survival setting. Stat Med. 2015;34(10):1659-80.

47. D'Agostino RB, Nam B-H. Evaluation of the performance of survival analysis models: discrimination and calibration measures. Advances in survival analysis; 2003. p. 1-25.

48. Miller ME, Langefeld CD, Tierney WM, Hui SL, McDonald CJ. Validation of probabilistic predictions. Med Decis Making. 1993;13(1):49-57.

49. Arkes HR, Dawson NV, Speroff T, Harrell FE Jr, Alzola C, Phillips $\mathrm{R}$, et al. The covariance decomposition of the probability 
score and its use in evaluating prognostic estimates. SUPPORT Investigators. Med Decis Making. 1995;15(2):120-31.

50. Steyerberg EW, Harrell FE Jr, Borsboom GJJM, Eijkemans MJC, Vergouwe Y, Habbema JDF. Internal validation of predictive models: Efficiency of some procedures for logistic regression analysis. J Clin Epidemiol. 2001;54(8):774-81.

51. R Core Team. R: a language and environment for statistical computing. Version 3.5.2 [software]. https://www.r-project.org/.

52. Goldstein DA, Chen Q, Ayer T, Chan KKW, Virik K, Hammerman A, et al. Bevacizumab for metastatic colorectal cancer: a global cost-effectiveness analysis. Oncologist. 2017;22(6):694-9.

53. Parikh RC, Du XL, Robert MO, Lairson DR. Cost-effectiveness of treatment sequences of chemotherapies and targeted biologics for elderly metastatic colorectal cancer patients. J Manag Care Spec Pharm. 2017;23(1):64-73.
54. Toumazis I, Kurt M, Toumazi A, Karacosta LG, Kwon C. Comparative effectiveness of up to three lines of chemotherapy treatment plans for metastatic colorectal cancer. MDM Policy Pract. 2017;2(2):2381468317729650.

55. Westwood M, van Asselt T, Ramaekers B, Whiting P, Joore M, Armstrong N, et al. KRAS mutation testing of tumours in adults with metastatic colorectal cancer: a systematic review and costeffectiveness analysis. Health Technol Assess. 2014;18(62).

56. Degeling K, Vu M, Koffijberg H, Wong HL, Koopman M, Gibbs P, IJzerman MJ. Health economic models for metastatic colorectal cancer: a methodological review. PharmacoEconomics. 2020;38:683-713.

\section{Affiliations}

\section{Koen Degeling ${ }^{1,2}\left(\mathbb{D} \cdot\right.$ Hui-Li Wong ${ }^{3,4}\left(\mathbb{C} \cdot\right.$ Hendrik Koffijberg $^{1}\left(\mathbb{C} \cdot\right.$ Azim Jalali $^{3} \cdot$ Jeremy Shapiro $^{5} \cdot$ Suzanne Kosmider $^{6}$. Rachel Wong ${ }^{3,7,8}$. Belinda Lee ${ }^{3,4,9} \cdot$ Matthew Burge ${ }^{10}$. Jeanne Tie ${ }^{3,4,6}$. Desmond Yip ${ }^{11}$. Louise Nott ${ }^{12}$.

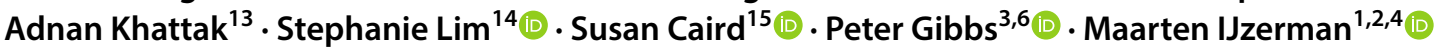

1 Health Technology and Services Research Department, Faculty of Behavioural, Management and Social Sciences, Technical Medical Centre, University of Twente, Enschede, The Netherlands

2 Cancer Health Services Research, School of Population and Global Health, Faculty of Medicine, Dentistry and Health Sciences, University of Melbourne, Melbourne, VIC, Australia

3 Personalised Oncology Division, Walter and Eliza Hall Institute of Medical Research, Melbourne, VIC, Australia

4 Department of Medical Oncology, Peter MacCallum Cancer Centre, Melbourne, VIC, Australia

5 Department of Medical Oncology, Cabrini Health, Melbourne, VIC, Australia

6 Department of Medical Oncology, Western Health, Melbourne, VIC, Australia

7 Department of Medical Oncology, Eastern Health, Melbourne, VIC, Australia
8 Eastern Health Clinical School, Monash University, Box Hill, VIC, Australia

9 Department of Medical Oncology, Northern Health, Melbourne, VIC, Australia

10 Department of Medical Oncology, Royal Brisbane and Women's Hospital, Brisbane, QLD, Australia

11 Department of Medical Oncology, The Canberra Hospital, Canberra, ACT, Australia

12 Department of Medical Oncology, Royal Hobart Hospital, Hobart, TAS, Australia

13 Department of Medical Oncology, Fiona Stanley Hospital, Perth, WA, Australia

14 Department of Medical Oncology, Campbelltown Hospital, Campbelltown, NSW, Australia

15 Department of Medical Oncology, Gold Coast University Hospital, Gold Coast, QLD, Australia 